Revue

Revue de l'histoire des religions

del'histoire des religions

4 | 2008

Varia

\title{
Jean BAUBÉROT, L’intégrisme républicain contre la laïcité
}

\section{Nicolas Piqué}

\section{OpenEdition \\ Journals}

Édition électronique

URL : http://journals.openedition.org/rhr/7043

DOI : $10.4000 /$ rhr.7043

ISSN : 2105-2573

Éditeur

Armand Colin

\section{Édition imprimée}

Date de publication : 1 décembre 2008

Pagination : 559-565

ISSN : 0035-1423

Référence électronique

Nicolas Piqué, "Jean вачвÉRot, L'intégrisme républicain contre la laïcité », Revue de l'histoire des religions [En ligne], 4 | 2008, mis en ligne le 15 janvier 2010, consulté le 22 septembre 2020. URL :

http://journals.openedition.org/rhr/7043 ; DOI : https://doi.org/10.4000/rhr.7043

Ce document a été généré automatiquement le 22 septembre 2020

Tous droits réservés 


\title{
Jean BAUBÉROT, L'intégrisme républicain contre la laïcité
}

\author{
Nicolas Piqué
}

\section{RÉFÉRENCE}

Jean BAUBÉROT, L'intégrisme républicain contre la laïcité, La Tour d'Aigues, Éditions de l'Aube, 2006, 302 p., $22 \mathrm{~cm}, 22 €$ (« Monde en cours »).

1 Le dernier livre de Jean Baubérot sur la laïcité tente de marier deux genres, deux livres, un premier développant des analyses « qui se veulent scientifiques » (p. 10) et un autre cherchant davantage à intervenir dans le débat citoyen, plus partisan. Le critère d'appréciation d'un tel projet en est d'autant plus compliqué.

On reconnaîtra dans un premier temps à cet ouvrage le mérite de présenter de manière claire et synthétique des analyses, pour partie déjà développées ailleurs et précédemment. C'est en particulier le cas des deuxième et troisième parties, consacrées respectivement aux «Impensés du centenaire de la loi de 1905 et leurs conséquences pour aujourd'hui » et à « Penser la laïcité ». Ce premier groupe d'analyses souligne avec profit la relative complexité de la période 1901-1908: la loi de 1905, son article 4 en particulier, a fait l'objet de négociations et de compromis dont l'enjeu est décisif pour l'auteur, distinguant entre un projet de laïcité intégral et celui qui sera voté, relevant davantage d'une conception libérale. La divergence du projet de Combes par rapport à celui de Briand-Pressensé-Jaurès est présentée dans son contexte mais aussi par rapport à sa signification actuelle. Car il convient, pour l'auteur, de savoir « comprendre le réel» (p. 145), qu'il s'agisse du réel historique, en ne faisant pas d'E. Combes l'auteur de la loi de 1905, ou du réel le plus contemporain, on y reviendra. Les analyses de cette deuxième partie se poursuivent en dénonçant les visions simplificatrices, voire fautives, parmi lesquelles figure l'exceptionnalité rattachée à la laïcité française. Là encore le recours à l'histoire, aux discours de Briand, permet de dissiper cette vision à la fois trop simple, trop rassurante et trop orgueilleuse. Ces 
rappels historiques permettent également à J. Baubérot de revenir, même rapidement, sur la notion de pacte laïque et ses enjeux, notion dont il fut le promoteur en 1990.

3 La troisième partie aborde la question laïque à partir de lieux plus circonscrits. D'abord en rappelant les termes grâce auxquels l'auteur a construit son analyse. S'ensuit une présentation, qu'on aurait souhaité plus étoffée, des enjeux d'une approche cherchant à dégager un "angle d'approche objectivant» (p. 201). Revenant sur l'histoire de ses propres recherches J. Baubérot précise comment il se distingue des chercheurs travaillant à partir de la notion de sécularisation : la situation particulière de la France, où c'est davantage «le politique, l'État qui définit l'espace légitime d'intervention de chaque institution » (p. 201), l'a amené à privilégier les notions de laïcité et laïcisation. Des références aux travaux de David Martin en particulier permettent de cerner la particularité du contexte historique et politique français. Deux questions sont ensuite traitées, qui permettent de mieux cerner toujours le contexte français, en examinant la pertinence de la notion de religion civile, puis en analysant les mutations contemporaines de la frontière entre public et privé. La première question donne lieu à un rappel historique soulignant combien la loi votée en 1905 ne comporte plus la dimension rousseauiste du projet de Combes; la comparaison avec les États-Unis permet ensuite à J. Baubérot de signaler les difficultés de la démocratie française à fonder ses valeurs, au-delà de ce que Jean-Paul Willaime appelle une "catho-laïcité ». L'analyse de la révolution touchant les «mutations du public et du privé », qui aboutit à un "désenchantement de la sécularisation » (p. 243), pour intéressante et décisive qu'elle soit reste bien allusive, reprenant en outre des recherches développées ailleurs par d'autres (on pense entre autre aux recherches proposées, dans le $n^{\circ} 87$ de la revue XXe siècle, par Denis Pelletier, Florence Rochefort et Dominique Memmi). L'ensemble de ces analyses ont toutefois l'indéniable avantage, à la fois, de resituer la laïcité dans la complexité de son histoire et de l'inscrire dans une réalité sociologique sans laquelle elle risque de n'être qu'un support idéologique. De plus cette présentation reste accessible, servie par un style sinon parlé, du moins vivant (fruit sans doute de l'origine de certains textes, présentés initialement sur le blog de l'auteur). L'ouvrage se termine par une quatrième partie présentant une Déclaration internationale sur la laïcité au XXIe siècle, dont les principes sont précisés dans le dernier chapitre, à partir de la notion de "laïcité inclusive", notion permettant à l'auteur de se distinguer non seulement des tenants d'une laïcité républicaine réputée abstraite, mais aussi des promoteurs d'une laïcité ouverte.

4 Les appréciations de la première partie ainsi que de l'avant-propos et de l'introduction sont loin de relever du même registre. Car le ton de l'auteur y est radicalement différent, n'échappant pas toujours à une expression dont l'aspect parfois virulent étonne dans le cadre d'un livre universitaire. L'auteur affiche pourtant la volonté de ne pas tomber dans l'invective pamphlétaire (p. 12), travers qu'il reproche souvent à ses adversaires (ces "brillants petits philosophes », p. 272, ces " petits maîtres penseurs " imposant leur "terrorisme intellectuel», p. 267), on aura compris les tenants d'un républicanisme universaliste. Même si les objets recensés et analysés dans cette première partie (les questions du genre, de l'Outre-Mer, des sectes et des rapports à l'islam), et choisis pour remettre en cause les "impensés de l'universalisme républicain " (p. 17), même si ces objets donc sont souvent analysés de manière intéressante et parfois novatrice (on pense en particulier aux analyses concernant les liens entre médecine et laïcité), il n'en demeure pas moins que la pertinence globale de ce début du livre reste, nous semble-t-il, obérée par des analyses dont la volonté de 
persuasion et le ton parfois cavalier à l'égard des contradicteurs brouillent finalement le propos.

5 En voici quelques exemples. La notion d'intégrisme tout d'abord; elle fait l'objet d'une présentation dans l'Introduction (p. 23) qui laisse sur sa faim le lecteur, même compte tenu de la prudence de l'auteur rappelant que le terme a un « usage scientifique très limité » (p. 23). L'élaboration qu'il en fait n'est pas totalement convaincante, renvoyant davantage à l'usage commun de dogmatisme combiné avec la dénonciation de ce que Hannah Arendt caractérise comme idéologie, i. e. une idée censée expliquer et rendre compte unilatéralement et exhaustivement de la réalité. En conséquence l'usage ultérieur qui en est fait, usage critique et dénonciateur du supposé intégrisme républicain, n'emporte pas vraiment l'adhésion. Autre exemple d'analyse contestable : pour souligner combien les représentations concernant la laïcité sont souvent caricaturales ou schématiques (cette critique salvatrice ouvrait déjà l'Introduction de l' Histoire de la laïcité en France, en opposant histoire et mémoire de la laïcité), J. Baubérot rapporte l'ignorance, ou l'oubli, d'étudiants américains de la devise « In God we trust » figurant sur les billets de un dollar, pour faire ensuite remarquer combien cette mention est abstraite, ayant peu d'impact et de sens au quotidien (p. 136). En revanche il s'arrête longuement sur la référence à l'Être suprême dans la Déclaration universelle des droits de l'homme (par ex. p. 227), supposée attester la permanence d'une conception proprement religieuse de la laïcité républicaine, alors que la même caractérisation d'abstraction aurait pu être retenue. Dernier exemple où la volonté de critiquer le modèle républicain universaliste l'emporte sur l'analyse nécessairement distanciée, lorsqu'est évoqué, dans le cadre du débat concernant le multiculturalisme (faut-il l'acclimater en France, constitue-t-il une caractéristique critiquable de la société anglaise ?), le «merveilleux modèle [français], tellement performant qu'il a conduit à la France à être la seule démocratie qui connaisse en 6 mois deux révoltes de sa jeunesse, celle des jeunes de banlieues en octobre-novembre 2005 et celle des jeunes de classes moyennes en mars-avril $2006 »$ (p. 114). La volonté de critique doit-elle conduire à assimiler deux révoltes (le terme est-il d'ailleurs légitime dans les deux cas ?) n'ayant absolument rien à voir entre elles ? On pourrait, ensuite, également faire remarquer que la révolte des banlieues de 2005 se produit 40 ans après celles, incroyablement plus violentes et meurtrières, de Watts aux États-Unis, et plus de vingt ans après celles de Brixton en Angleterre. Enfin qu'aurait-on entendu comme critique du modèle républicain si, à l'instar de ce qui s'est passé en Grande Bretagne, un terrorisme islamiste endogène s'était développé en France ? Évidemment la critique du modèle universaliste républicain est possible et légitime, comme le rappelle J. Baubérot (p. 267-268), mais à condition que la volonté polémiste aille de pair avec le sérieux revendiqué (p. 10).

6 Je souhaiterais terminer ce compte rendu par l'analyse de deux questions me paraissant au centre du désaccord opposant J. Baubérot aux tenants d'une conception républicaine de la laïcité. D'abord il me semble que cette opposition est tout autant disciplinaire qu'idéologique; elle tient beaucoup aux conceptions divergentes de la réalité que se font les sociologues et les philosophes. J. Baubérot le souligne à plusieurs reprises, sur un mode critique il est vrai : ses adversaires ne tiennent pas compte de la réalité (p. 22, p. 145), à tel point qu'il porte à leur encontre le diagnostic de « déni de la réalité » (p. 10). Mais, pour une tradition platonicienne de la philosophie, la réalité ne doit pas être confondue avec l'ensemble des faits. La laïcité est autant une idée qu'un ensemble de faits politiques, juridiques ou sociaux. C'est à cette aune que des philosophes (on peut 
penser aux travaux de Catherine Kintzler et de Henri Pena-Ruiz, mais aussi de Jacques Muglioni) ont pu défendre une conception idéelle sinon idéaliste de la laïcité. Le choix de cette option, renvoyant en l'occurrence en bonne part aux schèmes de Du Contrat Social, peut effectivement faire la part belle à l'imagination (comme l'analyse Pierre Bouretz dans La République et l'Universel, Gallimard, 2000), mais à une imagination créatrice, la même qui promeut l'idée d'égalité au-delà de toutes les inégalités factuelles que tout un chacun peut remarquer quotidiennement sans pour autant invalider l'idée d'égalité. Le déni de réalité est alors un reproche que l'on peut aussi, de manière inversée, adresser à J. Baubérot, au nom de l'oubli du caractère régulateur des principes, oubli donnant lieu à un factualisme libéral tout aussi critiquable. Le débat ne pourra avoir lieu que si les débatteurs acceptent de tenir compte de cette différence d'approche fondamentale. Dernier point, dernier lieu de désaccord, aperçu mais jamais vraiment discuté par l'auteur : la conception que l'on se fait du rapport entre individu, citoyen et État. La même remise en cause de la thèse universalisatrice rousseauiste, pour faire simple, se trouvait déjà au centre du dialogue entre Alain Renaut et Alain Touraine (Un débat sur la laïcité, Stock, 2005). Cette divergence me paraît décisive; J. Baubérot l'aborde, rapidement, lorsqu'il écrit que «L'État n'a pas à émanciper l'individu [...] c'est à l'individu de s'émanciper lui-même » (p. 173). Toutefois la critique de cette position républicaine émancipatrice relève davantage d'une attaque purement idéologique érigeant la réalité (factuelle, historique on aura compris) comme seul juge de paix. Ce débat aurait mérité d'autres développements, d'autant qu'ici encore les différents niveaux d'analyse sont confondus, comme si la logique philosophique républicaine pouvait être invalidée au nom d'une réalité factuelle qu'elle ne reconnaît pas toujours comme légitime (au risque de tomber dans l'argument pour le moins tendancieux consistant à opposer et reprocher à cette position républicaine le fait du subventionnement public de l'enseignement privé confessionnel, pp.138-139, alors même que les opposants de 1959 à la loi Debré l'ont précisément été au nom de cette logique républicaine). La discussion, parfaitement légitime, de ce présupposé universaliste ne pourra se faire que sur la base d'une confrontation d'idées, ce qui implique de ne pas taxer ses adversaires d'idéologues pour se réserver, seul, les mérites de la clairvoyance.

7 Le dernier mot du livre, «Et toc ! », même s'il n'en résume pas, on l'a dit, l'ensemble du ton, laisse encore entrevoir une conception agonistique du débat où il convient de clouer le bec de l'adversaire. Conception bien peu laïque du débat intellectuel on en conviendra, quel que soit d'ailleurs le type de laïcité auquel on se réfère.

\section{AUTEURS}

\section{NICOLAS PIQUÉ}

Université de Grenoble I (IUFM) 